\title{
Lower corneal nerve fibre length identifies diabetic neuropathy in older adults with diabetes: results from the Canadian Study of Longevity in Type 1 Diabetes
}

\author{
Daniel Scarr $^{1}$ - Leif E. Lovblom ${ }^{1}$ - Julie A. Lovshin ${ }^{2,3}$ • Geneviève Boulet ${ }^{1}$. \\ Mohammed A. Farooqi ${ }^{1}$ - Andrej Orszag ${ }^{1}$ - Alanna Weisman ${ }^{1,3}$ - Nancy Cardinez ${ }^{1}$.

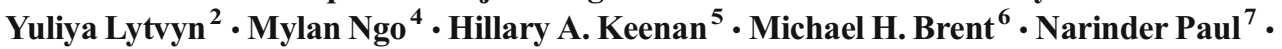 \\ Vera Bril $^{4}$ • David Z. I. Cherney ${ }^{2}$ - Bruce A. Perkins ${ }^{1,3}$
}

Received: 30 June 2017 / Accepted: 14 August 2017 / Published online: 3 October 2017

(C) Springer-Verlag GmbH Germany 2017

Keywords Corneal confocal microscopy · Corneal nerve fibre length $\cdot$ Diabetic neuropathy $\cdot$ Type 1 diabetes

$\begin{array}{ll}\text { Abbreviations } \\ \text { CNBD } & \text { Corneal nerve branch density } \\ \text { CNFD } & \text { Corneal nerve fibre density } \\ \text { CNFL } & \text { Corneal nerve fibre length } \\ \text { IVCCM } & \text { In vivo corneal confocal microscopy } \\ \text { ROC } & \text { Receiver operating characteristic } \\ \text { ROC-AUC } & \text { Area under the ROC curve }\end{array}$

Bruce A. Perkins

bruce.perkins@sinaihealthsystem.ca

1 Lunenfeld-Tanenbaum Research Institute, Leadership Sinai Centre for Diabetes, Mount Sinai Hospital, L5209-60 Murray Street Box 16, Toronto, ON M5T 3L9, Canada

2 Division of Nephrology, Department of Medicine, University of Toronto, Toronto, ON, Canada

3 Division of Endocrinology and Metabolism, Department of Medicine, University of Toronto, Toronto, ON, Canada

4 The Ellen and Martin Prosserman Centre for Neuromuscular Diseases, Krembil Neuroscience Centre, Division of Neurology, Department of Medicine, University Health Network, University of Toronto, Toronto, ON, Canada

5 Research Division, Joslin Diabetes Center, Boston, MA, USA

6 Department of Ophthalmology and Vision Sciences, Department of Medicine, University of Toronto, Toronto, ON, Canada

7 Joint Department of Medical Imaging, Division of Cardiothoracic Radiology, University Health Network, Toronto, ON, Canada
To the Editor: There exists an urgent need to better characterise and identify the presence of early-stage diabetic neuropathy when therapy is most likely to be effective. The lack of an objective endpoint for early neuropathy has seriously hindered the evaluation of disease-modifying therapies in clinical research and the prediction of neuropathy progression in clinical care $[1,2]$. There is considerable evidence that injury to small, thinly myelinated and unmyelinated nerve fibres precedes injury to large myelinated fibres in individuals with diabetic neuropathy [3].

Morphological examination of the small nerve fibres of the cornea by in vivo corneal confocal microscopy (IVCCM) has emerged as an objective and non-invasive imaging technique for identifying diabetic neuropathy. Specifically, lower corneal nerve fibre length (CNFL) has been confirmed as a valid biomarker for neuropathy identification in younger adults with type 1 diabetes [4, 5] and may represent a surrogate endpoint for trials of disease-modifying therapies for neuropathy. However, CNFL's diagnostic performance may be impaired with advanced age and diabetes duration owing to age- and extensive disease-related changes in corneal nerve morphology [6]. We aimed to determine whether CNFL retains its diagnostic validity in a unique cohort of older adults who have lived with type 1 diabetes for over 50 years.

As part of the second phase of the Canadian Study of Longevity in Type 1 Diabetes [7], 67/75 (89\%) participants with type 1 diabetes and 69/75 (92\%) participants forming a non-diabetic control group from age/sex-matched subgroups underwent electrophysiology-based procedures to define neuropathy (reference standard) and evaluation of corneal morphology by IVCCM (index test) in a cross-sectional analysis of the baseline evaluation. All participants provided written informed consent and the study and its procedures were approved by the institutional ethics board at the University 
Health Network and Mount Sinai Hospital in Toronto, ON, Canada. Based on consensus criteria, neuropathy was defined by (1) the presence of one or more neuropathic symptoms or signs; corroborated by (2) the presence of abnormality in one or more nerve conduction study variables in the sural and peroneal nerves. Abnormal nerve conduction study variables were defined as being $\leq 1$ st percentile or $\geq 99$ th percentile in a healthy population after adjustment for age and height. Participants underwent bilateral examination of the area adjacent to Bowman's layer of the cornea by IVCCM using the Rostock Cornea Module of the Heidelberg Tomograph III (Heidelberg Engineering, Smithfield, RI, USA) according to published methods [8]. The $400 \mu \mathrm{m}^{2}$ field-of-view lens was used. Measured variables were CNFL, corneal nerve branch density (CNBD) and corneal nerve fibre density (CNFD). Variables were measured using (1) a manual protocol (indicated by subscript MANUAL) where one image per eye was traced (CCMetrics v1.1, developed by M. Dabbah, University of Manchester, UK) and the variables were bilaterally averaged; and (2) a clinically generalisable automated protocol (indicated by subscript AUTO) where five-ten images per eye were rapidly analysed (ACCMetrics v2.0, developed by M. Dabbah and $\mathrm{X}$. Chen, University of Manchester, UK) and the images yielding the highest CNFL per eye were bilaterally averaged. General characteristics and IVCCM data are expressed as mean $\pm \mathrm{SD}$, median (interquartile range), or $n(\%)$. Comparisons were made using Student's $t$ test, Wilcoxon rank-sum test, or $\chi^{2}$-test. Receiver operating characteristic (ROC) curves were generated for each IVCCM variable and the area under the ROC curve (ROC-AUC) was used to determine diagnostic accuracy. ROC-
AUC was calculated using the trapezoidal rule and $95 \%$ CIs were calculated. The diagnostic accuracy of each test was compared with $\mathrm{CNFL}_{\text {MANUAL }}$ using the net reclassification index. The optimal threshold for identification of neuropathy of each variable was determined by finding the point on the ROC curve closest to the point of perfect discrimination using the formula $\sqrt{(0-x)^{2}+(1-y)^{2}}$. An $\alpha$-level of 0.05 (two-tailed) was used for tests of statistical significance. All analyses were performed using SAS 9.2 for Windows (SAS Institute, Cary, NC).

On average, individuals in the non-diabetic control group were $64 \pm 8$ years old and 39 (56\%) were female, while the participants with type 1 diabetes were $65 \pm 7$ years old $(p=0.49)$ and $36(54 \%)$ were female $(p=0.86)$. The participants with type 1 diabetes had a median duration of living with diabetes of $54(52-58)$ years, an $\mathrm{HbA}_{1 \mathrm{c}}$ of $7.3 \pm 0.8 \%(56 \pm 9 \mathrm{mmol} / \mathrm{mol})$ and $59(88 \%)$ met the case consensus criteria for neuropathy. Risk factors for neuropathy, such as duration of diabetes, $\mathrm{HbA}_{1 \mathrm{c}}$ level and cholesterol profile, were similar between participants with neuropathy (cases) and the neuropathy control group, although the control participants were shorter in height $(1.59 \pm 0.08$ vs $1.66 \pm 0.09 \mathrm{~m}$, $p=0.039)$ and had lower urinary albumin:creatinine ratios $(0.9$ [0.7-1.2] vs 1.7 [0.9-5.5] mg/mmol, $p=0.047$ ). Measurements of the IVCCM small nerve fibre variables and the corresponding performance metrics for neuropathy identification are presented in Table 1. For comparison, the non-diabetic control participants had mean $\mathrm{CNFL}_{\mathrm{MANUAL}}$ of $19.4 \pm 6.4$ and mean $\mathrm{CNFL}_{\mathrm{AUTO}}$ of $13.5 \pm 4.5 \mathrm{~mm} / \mathrm{mm}^{2}$. Compared with the neuropathy controls, neuropathy cases had lower $\mathrm{CNFL}_{\mathrm{MANUAL}}(10.5 \pm 5.6$ vs

Table 1 Baseline IVCCM measures for the 136 participants and ROC curve analysis for the concurrent identification of neuropathy in 67 participants with longstanding type 1 diabetes

\begin{tabular}{|c|c|c|c|c|c|c|c|c|c|}
\hline \multirow[t]{2}{*}{ Variable } & & \multirow{2}{*}{$\begin{array}{l}\text { Non-diabetic } \\
\text { controls }(n=69)\end{array}$} & \multicolumn{7}{|c|}{ Participants with type 1 diabetes $(n=67)$} \\
\hline & & & $\begin{array}{l}\text { Neuropathy } \\
\text { controls }(n=8)\end{array}$ & $\begin{array}{l}\text { Neuropathy cases } \\
(n=59)\end{array}$ & $\begin{array}{l}\text { ROC-AUC } \\
(95 \% \mathrm{CI})\end{array}$ & $\begin{array}{l}p \\
\text { value }^{\mathrm{a}}\end{array}$ & $\begin{array}{l}\text { Optimal } \\
\text { threshold }\end{array}$ & Sens & Spec \\
\hline $\mathrm{CNFL}_{\text {MANUAL }}$ & $\left(\mathrm{mm} / \mathrm{mm}^{2}\right)$ & $19.4 \pm 6.4$ & $17.9 \pm 7.0$ & $10.5 \pm 5.6$ & $0.81(0.66,0.95)$ & - & $\leq 13.7$ & 0.73 & 0.75 \\
\hline $\mathrm{CNFL}_{\text {AUTO }}$ & $\left(\mathrm{mm} / \mathrm{mm}^{2}\right)$ & $13.5 \pm 4.5$ & $12.2 \pm 5.6$ & $7.7 \pm 1.6$ & $0.80(0.67,0.93)$ & 0.27 & $\leq 10.7$ & 0.83 & 0.63 \\
\hline $\mathrm{CNFD}_{\text {MANUAL }}$ & (fibres $/ \mathrm{mm}^{2}$ ) & $25.7 \pm 9.2$ & $25.8 \pm 11.9$ & $15.4 \pm 7.2$ & $0.78(0.60,0.96)$ & 0.91 & $\leq 18.8$ & 0.76 & 0.75 \\
\hline $\mathrm{CNFD}_{\text {AUTO }}$ & (fibres $/ \mathrm{mm}^{2}$ ) & $19.7 \pm 9.3$ & $18.7 \pm 11.6$ & $8.4 \pm 7.9$ & $0.79(0.64,0.94)$ & 0.68 & $\leq 9.4$ & 0.66 & 0.75 \\
\hline $\mathrm{CNBD}_{\text {MANUAL }}$ & (branches $/ \mathrm{mm}^{2}$ ) & $57.0 \pm 34.0$ & $41.8 \pm 33.9$ & $25.6 \pm 20.7$ & $0.65(0.44,0.86)$ & 0.015 & $\leq 15.6$ & 0.44 & 0.75 \\
\hline $\mathrm{CNBD}_{\text {AUTO }}$ & (branches $/ \mathrm{mm}^{2}$ ) & $31.2 \pm 21.7$ & $27.7 \pm 18.5$ & $11.9 \pm 16.7$ & $0.76(0.57,0.95)$ & 0.11 & $\leq 9.4$ & 0.63 & 0.88 \\
\hline
\end{tabular}

Data are presented as mean $\pm \mathrm{SD}$

${ }^{a} p$ value for comparison of ROC-AUC to that of CNFL

The optimal threshold indicates the value used for case identification. The values for Sens and Spec correspond to each test's indicated optimal threshold All variables were lower across the non-diabetic controls, neuropathy controls, and neuropathy cases (ANOVA $p<0.001$ for each variable)

The 67 participants with type 1 diabetes had mean $\mathrm{CNFL}_{\mathrm{MANUAL}}$ of $11.4 \pm 6.2 \mathrm{~mm} / \mathrm{mm}^{2}$ and mean $\mathrm{CNFL}_{\mathrm{AUTO}}$ of $8.3 \pm 4.5 \mathrm{~mm} / \mathrm{mm}^{2}$ (irrespective of neuropathy status)

The ROC-AUCs of all IVCCM variables were not statistically different from those of $\mathrm{CNFL}_{\text {MANUAL }}(p>0.05$ for all comparisons), except for $\mathrm{CNBD}_{\text {MANUAL }}(p=0.015)$

Sens, sensitivity; Spec, specificity 
$\left.17.9 \pm 7.0 \mathrm{~mm} / \mathrm{mm}^{2}, p<0.001\right)$ and $\mathrm{CNFL}_{\mathrm{AUTO}}(7.7 \pm 1.6 \mathrm{vs}$ $\left.12.2 \pm 5.6 \mathrm{~mm} / \mathrm{mm}^{2}, p<0.001\right)$. CNFD ${ }_{\text {MANUAL }}, \mathrm{CNFD}_{\text {AUTO }}$, and $\mathrm{CNBD}_{\mathrm{AUTO}}$ were lower in neuropathy cases compared with the neuropathy controls $(p<0.05$ for all measures); $\mathrm{CNBD}_{\text {MANUAL }}$ tended to be lower in neuropathy cases compared with the neuropathy controls but this difference did not reach statistical significance $(p=0.061)$. $\mathrm{CNFL}_{\text {MANUAL }}$ had the highest ROC-AUC at 0.81 , with an optimal operating threshold of $\leq 13.7 \mathrm{~mm} / \mathrm{mm}^{2}$. This threshold had $73 \%$ sensitivity and $75 \%$ specificity, and had a positive and negative predictive value of $95 \%$ and $42 \%$, respectively. $\mathrm{CNFL}_{\mathrm{AUTO}}$ had a ROC-AUC of 0.80 and an optimal operating threshold of $\leq 10.7 \mathrm{~mm} / \mathrm{mm}^{2}$. This threshold had $83 \%$ sensitivity and $63 \%$ specificity, and had a positive and negative predictive value of $93 \%$ and $41 \%$, respectively.

Previous studies have defined the non-invasive, objective and rapid nature of the IVCCM procedure, established a clinically applicable automated image-analysis protocol and confirmed its potential to be performed by eye specialists during routine complication screening visits for individuals with diabetes. While there are a number of studies that have confirmed the diagnostic validity of CNFL for diabetic neuropathy identification in younger adults $[4,5]$, no studies to date have evaluated this imaging biomarker in older adults with diabetes. A key study [4] reported a ROC-AUC of 0.88 and an optimal threshold of $\leq 14.0 \mathrm{~mm} / \mathrm{mm}^{2}$ for $\mathrm{CNFL}_{\text {MANUAL }}$ in 89 participants with type 1 diabetes of mean age $41 \pm 16$ years, mean $\mathrm{CNFL}_{\text {MANUAL }}$ of $14.7 \mathrm{~mm} / \mathrm{mm}^{2}$ and median diabetes duration of $22(11-33)$ years. The current study yielded a ROC-AUC of 0.81 and a threshold value of $\leq 13.7 \mathrm{~mm} / \mathrm{mm}^{2}$ for $\mathrm{CNFL}_{\text {MANUAL }}$ despite the 67 participants with type 1 diabetes being substantially older (mean age $65 \pm 7$ years), with mean $\mathrm{CNFL}_{\text {MANUAL }}$ of $11.4 \mathrm{~mm} / \mathrm{mm}^{2}$ and living with diabetes for a longer duration (median 54 [52-58] years).

We confirmed that CNFL is a valid surrogate endpoint for the clinical and electrophysiological definition of diabetic neuropathy in older individuals. Specifically, measures of CNFL abnormality using manual and automated quantification methods were associated with the presence of neuropathy and CNFL was the optimal IVCCM variable. These findings confirm that age-related changes in CNFL morphology do not appear to impair diagnostic performance for the identification of neuropathy and similar CNFL screening protocols may be applicable to adult populations with type 1 diabetes over a broad age range. The validity of CNFL as a surrogate endpoint in an older population with type 1 diabetes raises substantial confidence in future work from our research group, together with collaborators (ClinicalTrials.gov Identifier NCT02423434), that aims to evaluate its use in disease-modifying intervention trials.

Acknowledgements This research was supported by Diabetes Canada and the JDRF Canadian Clinical Trial Network, as well as R. and J. Frisch and The Harvey and Annice Frisch Family Fund.
Data availability The datasets generated during and/or analysed during the current study are available from the corresponding author on reasonable request.

Funding This research was funded by the JDRF Canada (17-2013-312).

Duality of interest JAL has received speaker honoraria from Novo Nordisk, Merck Sharp \& Dohme, Eli Lilly, Intarcia Therapeutics and AstraZenca. GB has received speaker honoraria from Johnson \& Johnson. HAK has received support from Sanofi. NP has received support from Toshiba Medical. DZIC has received speaker honoraria from Janssen, AstraZeneca, Boehringer Ingelheim, Eli Lilly and Merck Sharp \& Dohme and has received research grant support from AstraZeneca, Merck Sharp \& Dohme and Boehringer Ingelheim. BAP has received speaker honoraria from Medtronic, Johnson \& Johnson, Roche, GlaxoSmithKline Canada, Novo Nordisk and Sanofi; has received research grant support from Medtronic and Boehringer Ingelheim; and serves as a consultant for NeuroMetrix.

Contribution statement All authors were involved in revising the manuscript critically for important intellectual content and for final approval of the version to be published. DS contributed to conception and design of the study, acquisition of data, analysis and interpretation of data and wrote the manuscript. LEL contributed to conception and design of the study and analysis and interpretation of the data. JAL, GB, MAF, $\mathrm{AO}$, and MN contributed to acquisition of data. AW, NC, YL, HAK, MHB, NP, and VB contributed to analysis and interpretation of data. DZIC and BAP contributed to conception and design of the study and analysis and interpretation of data. BAP is the guarantor of this work and, as such, had full access to all the data in the study and takes responsibility for the integrity and accuracy of the data and analysis.

\section{References}

1. Ziegler D, Luft D (2002) Clinical trials for drugs against diabetic neuropathy: can we combine scientific needs with clinical practicalities? Int Rev Neurobiol 50:431-463

2. Boulton AJ, Kempler P, Ametov A, Ziegler D (2013) Whither pathogenetic treatments for diabetic polyneuropathy? Diabetes Metab Res Rev 29:327-333

3. Breiner A, Lovblom LE, Perkins BA, Bril V (2014) Does the prevailing hypothesis that small-fiber dysfunction precedes largefiber dysfunction apply to type 1 diabetic patients? Diabetes Care 37:1418-1424

4. Ahmed A, Bril V, Orszag A et al (2012) Detection of diabetic sensorimotor polyneuropathy by corneal confocal microscopy in type 1 diabetes: a concurrent validity study. Diabetes Care 35:821-828

5. Tavakoli M, Quattrini C, Abbott C et al (2010) Corneal confocal microscopy: a novel noninvasive test to diagnose and stratify the severity of human diabetic neuropathy. Diabetes Care 33:1792-1797

6. Tavakoli M, Ferdousi M, Petropoulos IN et al (2015) Normative values for corneal nerve morphology assessed using corneal confocal microscopy: a multinational normative data set. Diabetes Care 38: 838-843

7. Bai JW, Lovblom LE, Cardinez M et al (2017) Neuropathy and presence of emotional distress and depression in longstanding diabetes: results from the Canadian study of longevity in type 1 diabetes. J Diabetes Complications 31:1318-1324

8. Tavakoli M, Malik RA (2011) Corneal confocal microscopy: a novel non-invasive technique to quantify small fibre pathology in peripheral neuropathies. J Vis Exp 3:2194 